\title{
What's in a Name? AIDS Dementia Complex, HIV-associated Dementia, HIV-associated neurocognitive disorder or HIV Encephalopathy
}

\author{
D Singh \\ HIV Prevention Unit, Medical Research Council of South Africa, Durban, South Africa
}

\begin{abstract}
The current paper reviews currently used and proposed nomenclature for neurocognitive disorders associated with HIV, and proposes a unitary system as well as recommends an operational approach to screening/diagnosing severe forms of HIV associated neurocognitive disorder (HAND) in order to identify individuals who might benefit from antiretrovirals (ARVs). The terms HIV dementia complex, HIV-associated dementia (HAD) and HIV encephalopathy (HIE) are being replaced by more refined definitions for the spectrum HIV associated neurocognitive disorder (HANDs). The Diagnostic and Statistical Manual (DSM) will introduce a further term- major neurocognitive disorder. The nosology can become very confusing as the terms are not exactly equivalent. Clinicians need guidance on how to interpret new terms to implement current legislation and treatment guidelines that use the old term HIE. As a WHO stage 4 disease, patients with HIE are eligible for ARVs irrespective of their CD4 count. However, there are no locally available operational criteria how to diagnose HIV encephalopathy (HIE). The updated terminology is preferred because it requires assessing cognition objectively with neuropsychological tests. It is recommended that the International HIV Dementia Scale be used to screen patients and to thereafter confirm diagnosis with further neuropsychological tests e.g. the trail making and digit span tests.
\end{abstract}

Key words: HIV; AIDS; HIV-associated dementia (HAD); HIV-associated neurocognitive disorder (HAND)

Received: 19-06-2011

Accepted: 01-08-201 1

doi: http://dx.doi.org/10.4314/ajpsy.v15i3.22

\section{Introduction}

The terms HIV dementia complex, HIV-associated dementia (HAD) and HIV encephalopathy (HIE) have been used interchangeably from the late 1980s to describe a clinical triad of cognitive, motor and behavioural changes typically seen in the advanced stages of HIV infection. ${ }^{1}$ The terms describing the spectrum of cognitive disorders in HIV positive patients have been revised from this clinical definition to more refined staging that require assessing specific neuropsychological domains. $2^{-5}$ Further changes in nosology are proposed by the Diagnostic and Statistical Manual (DSM) working group for DSM-5.

\section{Antiretroviral treatment}

In 1987, the Center for Disease Control and Prevention included HIV dementia as an AIDS-defining illness. The World Health Organization (WHO) included HIV encephalopathy in its case definition of stage four clinical disease. ${ }^{6}$ The WHO and the South African government's clinical guidelines for management of HIV recommend initiating ARVs in people with stage four disease irrespective of CD 4 count. ${ }^{7}$ As a WHO clinical stage four disease, patients with HIE are eligible for antiretrovirals (ARV) irrespective of their CD4 count in the private and public health sector. ${ }^{7}$ The Medical Schemes Act uses the term Prescribed Minimum benefit (PMB) to describe the set of benefits all members have access to certain minimum services irrespective of the benefit option they have selected. ${ }^{8} \mathrm{HIE}$ is one of 270 PMB conditions. As a PMB condition, patients are eligible for ARVs (and all relevant care). The South African HIV Clinician Society recommends starting ARVs when the CD4 is $<350 \mathrm{mmol} / \mathrm{l}$ and government has also revised their current 
recommendations to start ARVs when CD4 <350mmol/1. Medical aids have no obligation to provide ARVs if the CD4 is $>350 \mathrm{mmol} / \mathrm{l}$. Clinicians must use the definition of PMB conditions to make medical aids comply with the law. Failure to diagnose HIE, disadvantages patients who may otherwise benefit from ARVs. However, there are no guidelines how to diagnose HIE in the national ARV program. ${ }^{7}$ Clinicians need diagnostic tools and clarification how the new terminology relates to current legislation and treatment guidelines.

\section{HIV-associated neurocognitive disorders}

The spectrum of HIV-associated neurocognitive disorders were revised twice by the American Academy of Neurology. Since 1987, two further categories were added; in 1991 the American Academy of Neurology introduced the category of HIV-associated minor cognitive-motor deficit and in 2007, the National Institute of Mental Health (NIMH) and the National Institute of Neurological Diseases and Stroke revised the classification and added asymptomatic neurocognitive impairment (ANI). 4 The new term HIV associated neurocognitive disorder (HAND) describes the full spectrum of neurological disease starting from ANI, minor neurocognitive disorder (MND) and HAD. The new classification requires one to systematically assess the following domains (attention-information processing; language; abstraction- executive; complex motor; memory and sensory perceptual/motor skills). The assessment of these domains requires neuropsychological (NP) tests and thereafter to compare their performance in these NP tests against population based norms. There are additional requirements, such as the measurement of functional performance.

\section{DSIM-5}

In South Africa we use both the American Psychiatric Association's classification of mental disorders as defined in The Diagnostic and Statistical Manual of Mental Disorders (DSM) and the WHO International Classification of Diseases (ICD). The current proposal for the criteria to be used for neurocognitive disorders in DSM-5 has been published in a draft form. ${ }^{5}$ Just as the DSM-IV contained no specific criteria for HAND, the same is proposed for HAND in the DSM-5. There is no reference to the criteria that have been designated for HAND in neurology. If the currently proposed criteria for neurocognitive disorder are successfully promulgated, not only will the criteria for HAND be improperly depicted in an over-generalized fashion but also it will remain the case that there will be no specific criteria for HAND at all. The DSM-5 working group is recommending that HAD be subsumed under a new disorder to be termed "Major Neurocognitive Disorder". The term dementia has been dropped completely because it has stigmatizing connotations. Further, AAN criteria set requires that at least two neuropsychological domains be impaired, whereas the DSM-5 require that one NP domain be impaired. There are other subtle differences in definitions and one has to be an expert in the field to keep pace with changes (Table I). If the DSM-5 proposal is accepted, we shall minimize the importance of HAND and further add to the ever expanding nosology. We need to demystify these terms and give clear guidance to the clinician to practically implement current legislation and treatment guidelines.

\begin{tabular}{|c|c|c|}
\hline Criteria & American Academy of Neurologist criteria for HAD & $\begin{array}{l}\text { Diagnostic and Statistical Manual - } 5 \text { criteria for } \\
\text { major neurocognitive disorder }\end{array}$ \\
\hline Aetiology & Specific aetiology viz. HIV is identified & No specific aetiology is identified \\
\hline Diagnosis & $\begin{array}{l}\text { Diagnosis must be based upon report by the patient or a } \\
\text { knowledgeable informant or by observation by a clinician together } \\
\text { with deficits on standardized NP testing }\end{array}$ & $\begin{array}{l}\text { Diagnosis is made wholly by standardized NP } \\
\text { testing. Further collateral from informant, self report } \\
\text { or clinical observation are not necessary. }\end{array}$ \\
\hline Number of NP domains abnormal & Two NP domains be impaired & One NP domain must be impaired \\
\hline $\begin{array}{l}\text { Number and type of domain of } \\
\text { domains to be tested }\end{array}$ & $\begin{array}{l}\text { Seven domains - Verbal/language; attention/working memory; } \\
\text { abstraction/executive functioning; memory; speed of information } \\
\text { processing; sensory-perceptual, and motor skills }\end{array}$ & $\begin{array}{l}\text { Six domains - Attention, executive ability, memory, } \\
\text { language, visuoconstructional-perceptual ability, } \\
\text { and social cognition. }\end{array}$ \\
\hline Differences in domain & $\begin{array}{l}\text { Does not include social cognition (recognition of emotions, } \\
\text { theory of mind, behavioural regulation). } \\
\text { Information processing speed and motor skills are separate } \\
\text { domains }\end{array}$ & $\begin{array}{l}\text { Social cognition has to be affected. } \\
\text { Information processing speed and motor skills } \\
\text { separate domains } \\
\text { Not included }\end{array}$ \\
\hline Functional status & $\begin{array}{l}\text { Marked interference with any activities of daily living (e.g., work, } \\
\text { home life, or social activities - regardless of whether they interfere } \\
\text { with independence). }\end{array}$ & $\begin{array}{l}\text { Interference in activities of daily living must affect } \\
\text { independence. }\end{array}$ \\
\hline Exclusionary criteria & $\begin{array}{l}\text { No evidence of another, pre-existing cause for the dementia } \\
\text { (e.g., other CNS infection, CNS neoplasm, cerebrovascular } \\
\text { disease, and pre-existing neurologic disease). }\end{array}$ & $\begin{array}{l}\text { Deficits must not be attributable to another Axis I } \\
\text { psychiatric disorder (e.g., major depressive } \\
\text { disorder or schizophrenia). }\end{array}$ \\
\hline
\end{tabular}




\section{HIV-associated dementia (HAD)}

$\mathrm{HAD}$ occurs in approximately 10-15\% of all individuals with HIV/AIDS and is more common in late stages of infection. ${ }^{9}$ Less severe forms of HAND occurs in 30-60\% of people infected with HIV depending on disease stage. ${ }^{9-10}$ In South Africa, $23.5 \%$ of the people attending an ARV clinic in Cape Town had HAND. ${ }^{11}$ In the pre-HAART era, HAD was common and more severe. People with HAD died within 6 months. ${ }^{12}$ The introduction of HAART, led to a greater than $40 \%$ decline in the incidence of $\mathrm{HAD} \cdot{ }^{10,13-14}$ As yet there are no treatment guidelines for $\mathrm{HAD}$, but it is generally accepted HAART improves cognition. ${ }^{15}$ Whilst we await recommendations on specific ARV regimes or other treatments, we should initiate patients with HAD on ARVs. Given the burden of HAND and direct benefits of starting ARVs, there is an urgency to develop rapid screening tools to detect and monitor HAND. Our recommendations are not too different to Joska et al's recent publication. ${ }^{16}$ However, we clarify how to diagnose MND and $\mathrm{HAD}$ and implement it under current legislation.

$\mathrm{HAD}$ is a 'sub-cortical' dementia however, with ARVs and increased survival a mixed picture is becoming more common. Traditional cognitive screens e.g. the Mini Mental State Examination (MMSE) which were developed for 'cortical' dementia, has low sensitivity in this population. ${ }^{17-18}$ Several brief neuropsychological tests have been developed for clinical use to detect HAD. ${ }^{19-20}$ These include the Mental Alternation Test, the Executive Interview, HIV Dementia Scale (HDS), Modified HIV Dementia Scale and International and the International HIV Dementia Scale (IHDS). The IHDS is particularly useful because it is brief, culture neutral, requires no special equipment. It consists of four items: memory registration of four objects; motor speed that require rapid tapping of thumb and first digit of nondominant hand; speed of information processing and/or executive functioning measured by a three- position hand alternating sequence and memory recall of the four objects. At the recommended cut-off score of 10, it had $80 \%$ sensitivity when tested in Uganda. ${ }^{21}$ However it is far from ideal as it had lower sensitivity in two South African studies. ${ }^{22-23}$ The IHDS was derived from the HIV Dementia Scale. Memory- registration and recall items are the same as the IHDS. Other items are: psychomotor speed is measured by asking patient to write upper case letters of the alphabet, attention is measured by looking for anti-saccadic eye movement and time taken to complete copying a cube. The HIV Dementia Scale has demonstrated good sensitivity in our population. ${ }^{24}$ However this test is not ideal because observing the anti-saccadic eye movement requires training and is difficult for non neurologists to administer. ${ }^{21}$ Modified HIV dementia scale has been validated without the antisaccadic eye movement error task. The score has been pro- rated and use the cut off of $<7.5 / 12$. Significant variation in the quality of the cube copy item has been observed by ethnicity as well as educational level. This scale may therefore be less sensitive for minorities and individuals with less education and non western background. ${ }^{25} \mathrm{We}$ have no local data on the reliability of this test in our local population and we are therefore reserved about making further recommendations on the use of the modified HDS in our setting. Skinner et al tested the relative performance of the MMSE, HDS and IHDS ${ }^{26}$ At the recommended cut-off the positive predictive value of the IHDS, HDS and MMSE were $58.8,60.0$ and $40 \% .{ }^{26}$ None of these tests are ideal; however, the IHDS shows most promise for wide scale implementation in our setting.

\section{Screening tests}

Screening tests are only useful as a first stage in a two stage diagnostic process. Given the positive predictive value of the IHDS is about $60 \%$, it implies that in a further $40 \%$ of people screened positive do not have HAD. Hence patients screened positive need confirmatory NP tests. After completing the NP tests, results are compared against standardized population means to classify if a patient has normal, ANI, MND or HAD. A NP battery can take many hours to complete, require special equipment and specially trained professionals. Population norms for many NP tests cannot be generalized in our population because they are usually derived from educated, Western Caucasian populations. ${ }^{27}$ Singh et al recently published local population norms for two NP tests commonly used to assess NP functioning in HIV positive patients. ${ }^{28}$ The digit span ( $A$ and $B)$ and trail making ( $A$ and $B$ ) tests assess four of the six cognitive domains impaired in HANDS. These subtests take around 10-15 minutes to complete, require no special material other than a stop watch, paper and pencil. They can be administered by a lay counsellor or other staff after training and certification from a person experienced in its use. The concept of task shifting in mental health is being advocated as one solution to address the resource constraints in developing countries. ${ }^{29}$ The tests are culture neutral, however, patients must have some formal education to understand alphabets and numbers and have no visual or hearing impairment. These four subtests can be administered and compared against local population norms to more accurately classify HANDs. Patients can be classified into normal, asymptomatic neurocognitive impairment, minor neurocognitive disorder (MND) or HAD. The skills of neuropsychologists or psychiatrists can never be replaced by task shifting. Where these skills are available, referral must be made to confirm the diagnosis.

These test are impractical in people with no education, physical disabilities e.g. blindness, deafness are motor problems from amputation or strokes. Other tests, e.g. digit symbol coding test, Hopkins auditory verbal learning test, grooved peg board and colour trails 1 and 2 maybe preferable. ${ }^{16}$

\section{When to start ARVs}

Patients with HIE and functional impairment can be started on ARVs irrespective of CD4 count according to the current South African government's treatment guidelines. For patients in the private sector, all benefits are protected as PMB condition as defined in the Medical Schemes Act. Patients with MND and HAD will benefit from ARVs and should be started on ARVs. MND is a more subtle diagnosis and wholly dependent on NP testing. Hence the diagnosis must be clearly documented or confirmed by person familiar with the NP tests. Clinicians must take precautions to reduce false positives in performing these NP tests to limit undue exposure to ARVs. 


\section{Future research}

We need fewer terms and simplification of criteria. The simplicity of the CD4 count as a proxy measure of one's immunity is well understood by the lay public. The current criteria need to be translated into a group of tests that give a single summary measure of cognitive function. The WHO definition of HIE needs to be revised to incorporate advances in the field. The emphasis on NP testing creates urgency for researchers to gather local population norms for the various subtests.

\section{Conclusion}

Whist we await harmonization of terms in HIV cognitive disorders, there is a need to educate all practitioners on implementation of currently available legislation and treatment guidelines for HIE. The term HIE, is not entirely equivalent to the new definition of HAD by the AAN or major neuro-cognitive disorder in DSM-5. However, we should use the updated definitions and diagnostic criteria for $\mathrm{HAD}$ and MND to implement the current, terminology, in our national ARV roll out program. All HIV positive patients with CD4 > $350 \mathrm{~mm} / 1$ should be screened with the IHDS and those who are positive should have confirmatory NP testing e.g. trails (A and B) and digit span (forward and backward). More detailed testing and referral to psychiatrists should be done where resources are available. In keeping with legislation patients with HAD must be started on ARVs irrespective of CD4 count Patients with MND will benefit from ARVs, however, diagnosis and functional impairment must be clearly documented with confirmatory NP tests.

\section{References}

1. Brew BJ, Sidtis JJ, Rosenblum M, Price RW. AIDS dementia complex. J R Coll Physicians Lond 1988;22:140-4.

2. American Academy of Neurology. Nomenclature and research case definitions for neurological manifestations of human immunodeficiency virus type-1 (HIV-1) infection. Neurology 1991;41:778-85.

3. American Psychiatric Association. Diagnostic and Statistical Manual of Mental Disorders. Fourth Edition. Text Revision. DSM-IV TR. Arlington, VA. 2000

4. Antinori A, Arendt G, Becker JT, Becker JT, Brew B J, Byrd DA ,Cherner $M$, et al. Updated research nosology for HIV-associated neurocognitive disorders. Neurology 2007;69:1789-99.

5. Jeste D, Blacker D, Blazer D. Neurocognitive Disorders. A Proposal from the DSM-5 Neurocognitive Disorders Work Group. Arlington,

VA: American Psychiatric Association; 2010.

6. World Health Organization. WHO case definitions of HIV for surveillance and revised clinical staging and immunological classification of HIV-related disease in adults and children. Geneva: World Health Organization; 2007.

7. Department of Health of South Africa. Clinical Guidelines for the Management of HIV \& AIDS in Adults and Adolescents. Pretoria; 2010

8. Department of Health of South Africa. Medical Schemes Act. In: No 131 of 1998.

9. McArthur JC, Brew BJ. HIV-associated neurocognitive disorders: is there a hidden epidemic? AIDS 2010;24:1367-70.

10. Grant I. Neurocognitive disturbances in HIV 1. Int Rev Psychiatry 2008:20:33-47

11. Joska JA, Fincham DS, Stein DJ, Paul RH, Seedat S. Clinical correlates of HIV-associated neurocognitive disorders in South Africa. AIDS

Behav 2010;14:371-8.

12. Brodt HR, Kamps BS, Gute P, Knupp B, Staszewski S, Helm EB Changing incidence of AIDS-defining illnesses in the era of antiretroviral combination therapy 1. AIDS 1997;1 1:1731-8.

13. McArthur JC. HIV dementia: an evolving disease. Journal of Neuroimmunology 2004;157:3-10

14. Sacktor N, MCDermott MP, Marder K, Schifitto G, Selnes OA, McArthur J C, et al. HIV-associated cognitive impairment before and after the advent of combination therapy. J Neurovirol 2002:8:136-42.

15. Joska JA, Gouse H, Paul RH, Stein DJ, Flisher AJ. Does highly active antiretroviral therapy improve neurocognitive function? A systematic review. J Neurovirol 2010;16:101-14.

16. Joska JA, Hoare J, Stein DJ, Flisher AJ. The neurobiology of HIV dementia: implications for practice in South Africa. Afr J Psychiatry 2011;14:17-22.

17. Butters N, Grant I, Haxby J,Judd, L, Martin A, MCClelland J, et al. Assessment of AIDS-related cognitive changes: recommendations of the NIMH Workshop on Neuropsychological Assessment . J Clin Exp Neuropsychol 1990;12:963-78.

18. Singh D. Neurocognitive impairment in PLWHA: clinical features and assessment. Southern African Journal of HIV Medicine 2009:30-4.

19. Power C, Selnes OA, Grim JA, McArthur JC. HIV Dementia Scale: a rapid screening test. JAcquir Immune DeficSyndr Hum Retrovirol 1995;8:273-8.

20. Jones BN, Teng EL, Folstein MF, Harrison KS. A new bedside test of cognition for patients with HIV infection. AnnInternMed 1993;119:1001-4

21. Sacktor NC, Wong M, Nakasujja N, Skolasky R, Selnes O, Musisi S, et al. The International HIV Dementia Scale: a new rapid screening test for HIV dementia. AIDS 2005; 19:1367-74.

22. Joska JA, Westgarth-Taylor J, Hoare J, Thomas K, Paul R, Myer L, et al. Validity of the International HIV Dementia Scale in South Africa. AIDS Patient Care STDS 2011;25:95-101.

23. Singh D, Goodkin K, Hardy DJ, Lopez E, Morales G. Diagnostic utility of the International HIV Dementia Scale for asymptomatic HIVassociated neurocognitive impairment and HIV-associated neurocognitive disorder in South Africa. . In: 18th International AIDS Conference; $201018-23$ July 2010; Vienna, Austria. ; 2010.

24. Ganasen KA, Fincham D, Smit J, Seedat S, Stein D. Utility of the HIV Dementia Scale (HDS) in identifying HIV dementia in a South African sample. JNeurol Sci 2008;269:62-4.

25. Davis HF, Skolasky RL, Jr., Selnes OA, Burgess DM, McArthur JC Assessing HIV-associated dementia: modified HIV dementia scale versus the Grooved Pegboard. AIDS Read 2002;12:29-31.

26. Skinner S, Adewale AJ, DeBlock L, Gill MJ, Power C. Neurocognitive screening tools in HIVIAIDS: comparative performance among patients exposed to antiretroviral therapy. HIV Med 2009; 10:246-52.

27. Lu L, Bigler ED. Normative data on trail making test for neurologically normal, Chinese-speaking adults. . Applied Neuropsychology 2002;9:219-25.

28. Singh D, Joska JA, Goodkin K. Normative scores for a brief neuropsychological battery for the detection of HIV-associated neurocognitive disorder (HAND) among South Africans. BMC Res Notes 2010;3:28.

29. Petersen I, Lund C, Bhana A, Flisher AJ. A task shifting approach to primary mental health care for adults in South Africa: human resource requirements and costs for rural settings. Health Policy Plan 2012;27(1):42-51. 